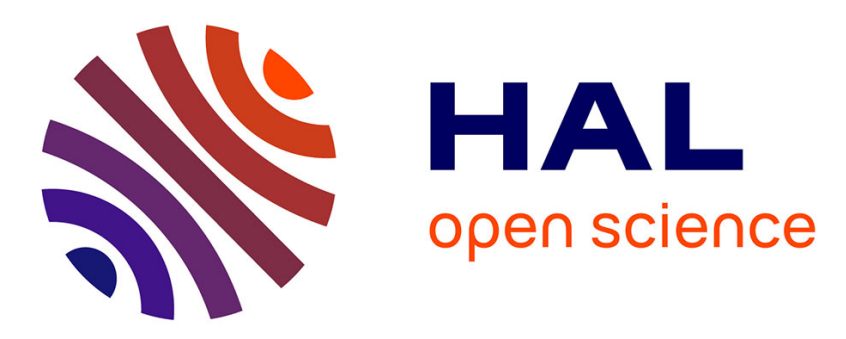

\title{
Simulation of large-scale periodic circuits by a homogenization method
}

\author{
Nicolas Ratier, Michel Lenczner
}

\section{To cite this version:}

Nicolas Ratier, Michel Lenczner. Simulation of large-scale periodic circuits by a homogenization method. Thermal, Mechanical and Multi-Physics simulation and Experiments in Microelectronics and Microsystems (EuroSimE 2009), Jan 2009, Delft, Netherlands. pp.1-6, 10.1109/ESIME.2009.4938413 . hal-00957614

\section{HAL Id: hal-00957614 https://hal.science/hal-00957614}

Submitted on 15 Apr 2021

HAL is a multi-disciplinary open access archive for the deposit and dissemination of scientific research documents, whether they are published or not. The documents may come from teaching and research institutions in France or abroad, or from public or private research centers.
L'archive ouverte pluridisciplinaire HAL, est destinée au dépôt et à la diffusion de documents scientifiques de niveau recherche, publiés ou non, émanant des établissements d'enseignement et de recherche français ou étrangers, des laboratoires publics ou privés. 


\title{
Simulation of Large-Scale Periodic Circuits by a Homogenization Method
}

\author{
N. Ratier, M. Lenczner \\ FEMTO-ST Institut, Time-Frequency department \\ 32 avenue de l'Observatoire, 25044 Besançon Cedex, France \\ nicolas.ratier@femto-st.fr, michel.lenczner@utbm.fr
}

\begin{abstract}
Actuation, sensoring and control in arrays of MEMS require spatially distributed periodic electronic circuits. For very large sized arrays, say $1000 \times 1000$ MEMS or cells, on the same chip, simulation requirements for electronics are far away from standard algorithm capabilities. One of the authors has shown in a theoretical paper [2], that a homogenization modeling method, previously developed for composite materials, can be extended to arrays of electronic circuits, at least in the linear static case.

When it is applied to a set of periodic network equations, the simplified resulting model turns to be a system of few partial differential equations. Its properties are inherited on the one hand from the periodic cell composition, and on the other hand from electric conditions imposed at the boundaries. Its numerical solution, a vector of few mean voltages, is weakly dependent of the array size. Actual voltages, at all nodes of the whole periodic circuit, are computed through a fast post-processing procedure. We present the implementation of the model.
\end{abstract}

\section{Introduction}

A tremendous progress in collective micro-fabrication processes has made possible the massive integration of Micro Electro Mechanical Systems (MEMS) on a single substrate. At present, there is a need to developp an efficient tool in terms of CPU time to simulate a very large array. This paper focuses on the simulation of spatially periodic circuits. The periodic unit cell is limited to linear and static components but its number can be very large. The theory presented here allows one to simulate an array of electronic circuits which are far away from the possibility of a regular circuit simulator like Spice. Our approch is based on the so-called two scale transform [2].

This paper presents a method that reformulates the electrical network equations in terms of partial differential equations (PDE). The numerical resolution of this PDE is straighforward and independent of the number of cells. Soving PDE and postprocessing its solution leads to an approximation of all voltages and currents. Theoretically, more the number of cells is large, more the model is accurate. The method is illustrated on a basic circuit to allow hand calculations, which are mostly matrix multiplications. Nevertheless, if the reader really wants to try the example, the authors strongly advise him to use rather a computer algebra software.

\section{Linear Static Periodic Circuits}

We consider the class of periodic circuits in $d$ space dimensions. An example of such circuit in two space dimensions is shown in Fig. 1. The circuit cell is detailled on Fig .2. Voltage or current sources, whose value may be zero, can be placed on the boundary to realize specific boundary conditions. We assume that the number of cells is large in all the $d$ directions. Mathematically, it is easier to formalize the problem by considering that the whole circuit occupies a unit square $\Omega=(0,1)^{d}$ and that the period lengths, in all directions, are equal to an identical small parameter $\varepsilon$ (Fig. 1).

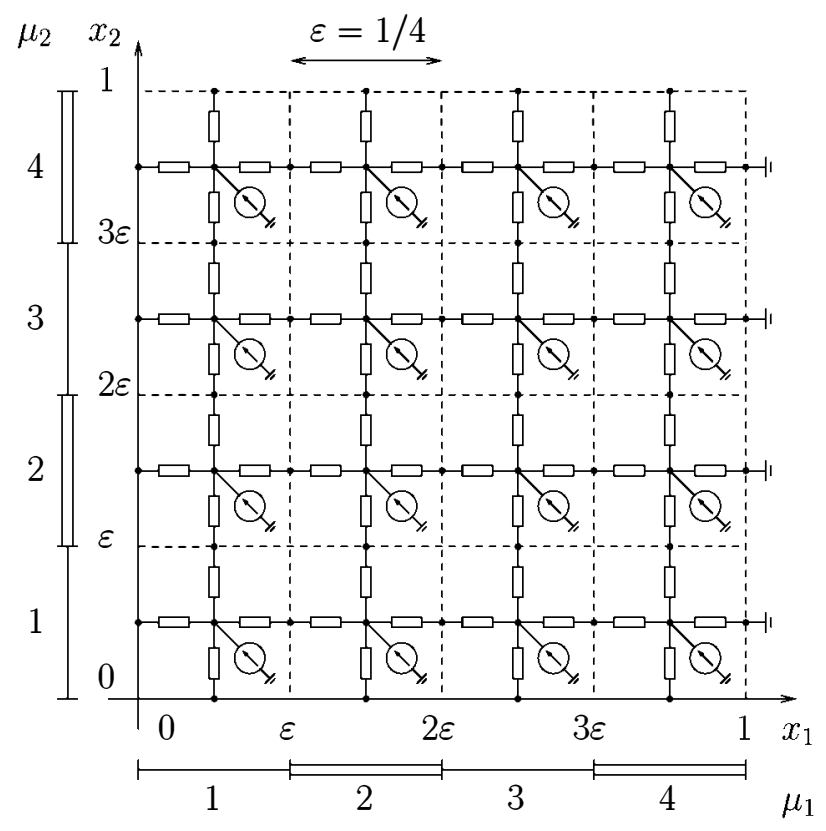

Fig. 1: Circuit example.

We limit ourselves to the study of circuits whose cell is linear and static. Precisely, the components of a cell are limited to the Spice elements R, V, I, E, F, G, H. All ports of any multiport components $\mathrm{E}, \mathrm{F}, \mathrm{G}, \mathrm{H}$ must belong to a same cell. The expanded cell is arbitrarily defined in a unit cell $Y=(-1 / 2,+1 / 2)^{d}$ (see Fig. 2). We map any discrete node $n$ onto the continuous coordinate $\left(y_{1}, \ldots, y_{d}\right)$. The vector $y(n) \in \mathbb{R}^{d}$ is the coordinate vector of a node $n$. For example, the coordinates of the nodes in Fig. 2 are $y(1, \ldots, 6)$, In particular, the coordinates of the node $n=3$ is the vector $(1 / 2,0)^{T}$.

$$
\begin{aligned}
& y(1, \ldots, 6) \\
= & \left(\begin{array}{cccccc}
-1 / 2 & 0 & 1 / 2 & 0 & 0 & 1 / 4 \\
0 & 0 & 0 & 1 / 2 & -1 / 2 & -1 / 4
\end{array}\right) .
\end{aligned}
$$

The maps of voltages and currents from the whole circuit (global network) to the cell circuit (local network) are defined as follows. First, we denote by 


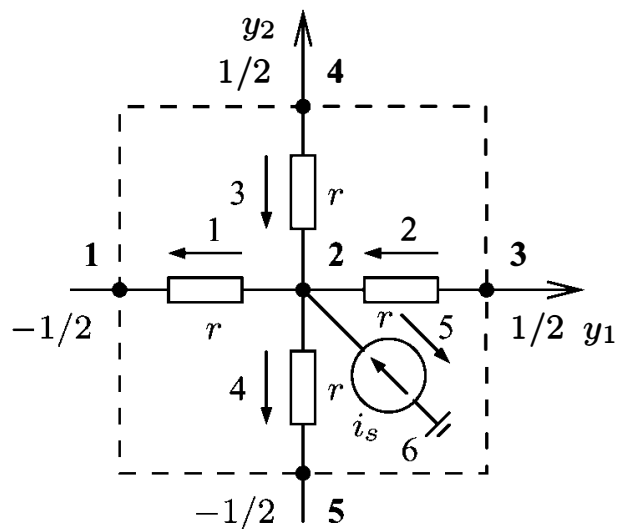

Fig. 2: Expanded cell of the circuit.

$\mathcal{E}=$ the branch set of the whole circuit,

$\mathcal{N}=$ the node set of the whole circuit,

$E=$ the branch set of the cell circuit,

$N=$ the node set of the cell circuit,

and we define three indices

- The global index $\mathcal{I}$ references all the branches of the whole circuit.

- The multi-integer $\mu=\left(\mu_{1}, . ., \mu_{d}\right) \in\{1, . ., m\}^{d}$ enumerates all the cells $Y_{\mu}^{\varepsilon}$ in the circuit $\Omega$.

- The local index $j \in\{1, . .|E|\}$ enumerates all the branches of the unit cell $Y$.

Each branch voltage or current can then be referenced by the index $\mathcal{I}$ or the couple $(\mu, j)$. This is a one-to-one correspondence denoted by $\mathcal{I} \sim(\mu, j)$. Using this correspondence, for each vector $\mathbf{u} \in \mathbb{R}^{|\mathcal{E}|}$ one may define a unique tensor $U_{\mu j}$ with $(\mu, j) \in\{1, . ., m\}^{d} \times\{1, . .,|E|\}$ by $U_{\mu j}=\mathbf{u}_{\mathcal{I}}$ for $(\mu, j) \sim \mathcal{I}$.

\section{Circuit Equations}

The electrical state of a circuit can be charaterized [1] by the vectors $(\varphi, \mathbf{v}, \mathbf{i})$ where,

$$
\begin{aligned}
& \varphi \in \mathbb{R}^{|\mathcal{N}|}=\text { the nodal voltages (or electric potentials), } \\
& \mathbf{v} \in \mathbb{R}^{|\mathcal{E}|}=\text { the branch voltages, } \\
& \mathbf{i} \in \mathbb{R}^{|\mathcal{E}|}=\text { the branch currents. }
\end{aligned}
$$

We can formulate the circuit equations under the form (1-4) where $\mathbf{u}_{s} \in \mathbb{R}^{|\mathcal{E}|}$ represents voltage and current sources merged in single vector completed by some zeros. Eq. (1) is the Kirchhoff's Voltage Law. Eq. (2) represents the constitutive equations and Eqs. $(3,4)$ correspond to the Tellegen theorem,

$$
\begin{aligned}
\mathbf{v} & =\mathcal{A}^{T} \boldsymbol{\varphi} \\
\mathcal{R} \mathbf{i}+\mathcal{M} \mathbf{v} & =\mathbf{u}_{s} \\
\mathbf{i}^{T} \mathbf{w} & =0 \\
\text { for all } \mathbf{w} & =\mathcal{A}^{T} \psi \text { with } \psi \in \Psi
\end{aligned}
$$

Here $\Psi$ is the set of admissible potentials for the circuit problem, that is to say:

$$
\begin{aligned}
\Psi= & \left\{\psi \in \mathbb{R}^{|\mathcal{N}|} \text { such that } \psi_{\mathcal{I}}=0\right. \\
& \text { for all ground nodes } \left.n_{\mathcal{I}}\right\} .
\end{aligned}
$$

Since the matrices $\mathcal{M} \in \mathbb{R}^{|\mathcal{E}|} \times \mathbb{R}^{|\mathcal{E}|}, \mathcal{R} \in \mathbb{R}^{|\mathcal{E}|} \times \mathbb{R}^{|\mathcal{E}|}$ and the vector $\mathbf{u}_{s} \in \mathbb{R}^{|\mathcal{E}|}$ are exclusively deduced from the branch equations of the circuit, they can be expressed in terms of two reduced matrices $M \in \mathbb{R}^{|E|} \times \mathbb{R}^{|E|}$ and $R \in \mathbb{R}^{|E|} \times \mathbb{R}^{|E|}$ and a reduced vector $u_{s} \in \mathbb{R}^{|E|}$. The reduced matrices and vector are simply derived from the constitutive equations of the unit cell, which are in the example,

$$
\begin{aligned}
-v_{1}+r i_{1} & =0, \\
-v_{2}+r i_{2} & =0, \\
-v_{3}+r i_{3} & =0, \\
-v_{4}+r i_{4} & =0, \\
i_{5} & =i_{s} .
\end{aligned}
$$

The transpose $\mathcal{A}^{T} \in \mathbb{R}^{|\mathcal{E}|} \times \mathbb{R}^{|\mathcal{N}|}$ of the incidence matrix can also be expressed in terms of a reduced matrix noted by $A^{T}$ (with a little abuse of notation). Notice that we cannot find a reduced matrix for the incidence matrix itself. We introduce the local (complete) incidence matrix $A \in \mathbb{R}^{|N|} \times \mathbb{R}^{|E|}$,

$$
A_{i j}= \begin{cases}+1 & \text { if } \operatorname{branch} j \text { leaves node } i \\ -1 & \text { if branch } j \text { enters node } i \\ 0 & \text { if branch } j \text { does not touch node } i\end{cases}
$$

The solution of the simplified model introduced in this paper realizes an approximation of the solution of $(1-4)$ for small values of $\varepsilon(\varepsilon<<1)$. It is derived as a limit of the latter when the cells length $\varepsilon$ diminishes towards zero.

\section{Direct Two-scale Transform $T_{E}$}

The general idea of the two-scale transform rests on gathering the voltages (or currents) denoted by $\widehat{\mathbf{u}}$ of a same branch $j$ of all cells. Indeed, the voltages (or currents) are defined by a function $\widehat{\mathbf{u}}_{j}(x)$, which depends on the parameter $\varepsilon$ and whose limit when $\varepsilon \rightarrow 0$ will be calculated.

Let us first denote by $\chi_{Y_{\mu}^{\varepsilon}}(x)$ the characteristic function of the cell $Y_{\mu}^{\varepsilon}$ equal to 1 when $x \in Y_{\mu}^{\varepsilon}$ and 0 otherwise. As an exemple, the characteristic function $\chi_{Y_{32}^{\varepsilon}}$ of the cell $\mu=(3,2)$ is represented in Fig. 3 .

The two-scale transform $\widehat{\mathbf{u}}$ of the vector $\mathbf{u} \in \mathbb{R}^{|\mathcal{E}|}$ belong to the set $\mathbb{P}^{0}(\Omega)^{|E|}$ of vectors $Y_{\mu}^{\varepsilon}$-piecewise constant of functions defined by (6) where $U_{\mu j}=\mathbf{u}_{\mathcal{I}}$ with $(\mu, j) \sim \mathcal{I}$,

$$
\widehat{\mathbf{u}}_{j}(x)=\sum_{\mu \in\{1, . ., m\}^{d}} \chi_{Y_{\mu}^{\epsilon}}(x) U_{\mu j} .
$$

For example, $\widehat{\mathbf{v}}_{j}(x)$ is the voltage $V_{\mu j}$ of the branch referred by the local index $j$ of the cell $\mu$ when $x$ belongs. By construction, the function $x \mapsto \widehat{\mathbf{v}}_{j}(x)$ is constant on all cells. Figure 4 illustrates this concept by representing the 


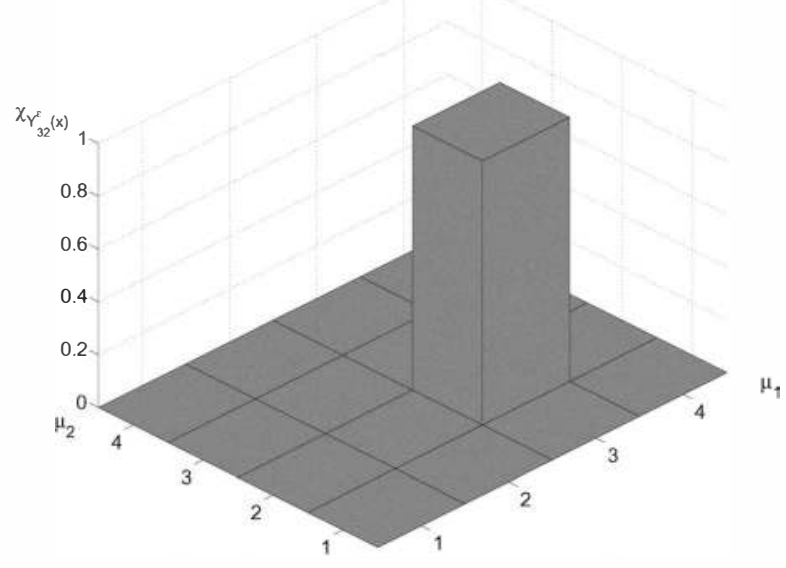

Fig. 3: Caracteristic Function $\chi_{Y_{32}^{\varepsilon}}$.

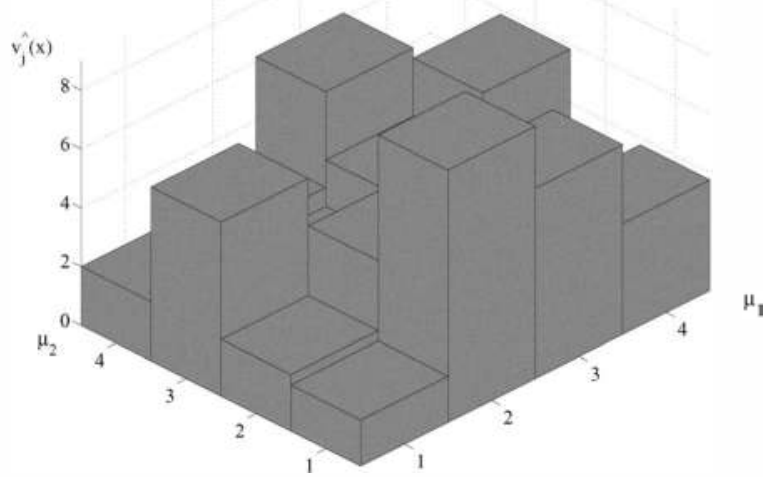

Fig. 4: One component $\widehat{\mathbf{v}}_{2}(x)$ of a two-scale transform.

components $\widehat{\mathbf{v}}_{2}(x)$. It indicates that the voltage $\widehat{\mathbf{v}}_{2}(x)$ of the branch $\mathrm{j}=2$ (cf. Fig. 2) of the cell $\left(\mu_{1}, \mu_{2}\right)=(1,4)$ is equal to $2 \mathrm{~V}$.

The two-scale transform $T_{E}$ of $\mathbf{u}$ is the linear map $\mathbf{u} \mapsto$ $\widehat{\mathbf{u}}$ from $\mathbb{R}^{|\mathcal{E}|}$ to $\mathbb{P}(\Omega)^{|E|} \subset L^{2}(\Omega)^{|E|}$. The model is derived from the limit when $\varepsilon \rightarrow 0$ of all vectors involved in the circuit equations. The actual circuit voltages and currents are finally computed by inverting the two-scale transform with the physical value of $\varepsilon$. Next Section is devoted the construction of $T_{E}^{-1}$.

\section{Inverse Two-scale Transform $T_{E}^{-1}$}

The calculation of the inverse two-scale transform $T_{E}^{-1}$ is done by computing the adjoint $T_{E}^{*}$ and then proving two identities properties beetween these transforms.

Let us recall that the norm of a vector in a general vector space is a generalization of the idea of the length of a vector. The inner product has been defined in the hope of extending the concept of angles between vectors. The inner product and the norm in $\mathbb{R}^{|\mathcal{E}|}$ and $L^{2}(\Omega)^{|E|}$ are denoted in the following table,

\begin{tabular}{|lll|}
\hline & $\mathbf{u}, \mathbf{v} \in \mathbb{R}^{|\mathcal{E}|}$ & $u, v \in L^{2}(\Omega)^{|E|}$ \\
\hline Inner product & $\frac{u, \mathbf{v}]}{(u, v)}$ \\
\hline Norm & $|\mathbf{v}|=[\mathbf{v}, \mathbf{v}]^{1 / 2}$ & $\|u\|=(u, u)^{1 / 2}$ \\
\hline
\end{tabular}

and defined by

$$
\begin{aligned}
{[\mathbf{u}, \mathbf{v}] } & =\varepsilon^{d} \mathbf{u}^{T} \cdot \mathbf{v} \\
(u, v) & =\sum_{j=1}^{|E|} \int_{\Omega} u_{j}(x) v_{j}(x) d x
\end{aligned}
$$

For all $u \in L^{2}(\Omega)^{|E|}$ and $v \in \mathbb{R}^{|\mathcal{E}|}$, the adjoint $T_{E}^{*} u$ is defined through the equality

$$
\left[T_{E}^{*} u, v\right]=\left(u, T_{E} v\right)
$$

The calculation of $T_{E}^{*}$ from (9) is given in Appendix and leads to

$$
\left(T_{E}^{*} u\right)_{\mathcal{I}}=\varepsilon^{-d} \int_{Y_{\mu}^{e}} u_{j}(x) d x
$$

Moreover, Appendix proves that $T_{E}^{*} T_{E}=I_{\mathcal{E}}$ on $\mathbb{R}^{|\mathcal{E}|}$ and $T_{E} T_{E}^{*}=I_{E}$ on $\mathbb{P}^{0}(\Omega)^{|E|}$. As $T_{E}$ is one-to-one from $\mathbb{R}^{|\mathcal{E}|}$ to $\mathbb{P}^{0}(\Omega)^{|E|}$, these two identities show that $T_{E}^{*}$ is its inverse

$$
T_{E}^{-1}=T_{E}^{*}
$$

\section{Behavior of "Spread" Analog Circuits}

A circuit spread out over a large region may have some pathes linking oposite sides. In view of deriving a partial differential equation for the electric potential, we assume that voltages are increments of the order $\varepsilon$ along such pathes. Flowing currents result of numerous $(1 / \varepsilon)$ additive sources coming from crossed cell contributions. Since they may converge when $\varepsilon$ vanishes the crossing current must be of magnitude 1 , and sources of the order of $\varepsilon$.

A branch which does not belongs to any crossing path is necessarily part of a path to the ground, so its voltage magnitude is 1 . We choose its magnitude current be of the order $\varepsilon$ as it may be a crossing path source. This assumption is not restrictive since we can choose an appropriate scaling law for its component.

The periodicity of the circuit implies that each node $n$ located on the boundary of the cell has its counterpart $n^{\prime}$ on the opposite side. We assume that each such couple is linked by at least a crossing path. We introduce the set $E_{C} \subset E$ constituted of all the branches of at least one path linking each couple $\left(n, n^{\prime}\right)$. Of course, a link between $\left(n, n^{\prime}\right)$ which includes a ground node is not considered as a path. The complementary set $E-E_{C}$ is denoted by $E_{N C}$ (non-crossing pathes). In the case where many crossing pathes are linking $n$ and $n^{\prime}$, the designer is free to decide which are included in $E_{C}$ and which are not, with regard to the above discussion about current and voltage magnitudes.

The subset $E_{C}$ is partitioned in its $n_{c}$ connected components $E_{C}=\cup_{p=1}^{n_{c}} E_{C p}$. In the following, the main result on the circuit equations will be derived for the connected components of $E_{C}$ and not for $E_{C}$ itself. 


\section{Cell Equations (Problem Micro )}

The model formulation is decomposed in four parts. Theorem 1, formulates the linear relation between mean electric potentials $\varphi_{C}^{0}$ along crossing pathes and the other fields as branch currents and voltages. This relation is strictly local in each cell. In the next Section, the linear relation is simply rewritten introducing linear operators. They are used in Theorem 2 for coefficients of the boundary value problem on $\varphi_{C}^{0}$. Finally, actual voltages and currents are computed thanks to the inverse two-scale transform.

The previous assumptions about voltage and current magnitudes is formulated using the scaling matrices $S_{v}, S_{c}$ and $S_{s}$ applied to the two-scale transforms,

$$
\begin{aligned}
\widehat{\mathbf{i}}^{\varepsilon} & =S_{c} \widehat{\hat{\mathbf{i}}}, \\
\widehat{\mathbf{v}}^{\varepsilon} & =S_{v} \widehat{\mathbf{v}}, \\
\widehat{\mathbf{u}}_{s}^{\varepsilon} & =S_{s} \widehat{\mathbf{u}}_{s} .
\end{aligned}
$$

with the $|E| \times|E|$ scaling matrices defined as

$$
\begin{aligned}
S_{v} & =\varepsilon^{-1} I_{E_{C}}+I_{E_{N C}}, \\
S_{c} & =I_{E_{C}}+\varepsilon^{-1} I_{E_{N C}}, \\
S_{s} & =\Pi_{c} S_{c}+\Pi_{v} S_{v} .
\end{aligned}
$$

Here the $|E| \times|E|$ matrices $I_{E_{C}}$ and $I_{E_{N C}}$ are the vector sub-spaces $\mathbb{R}^{|E|}$ generated by non vanishing values on $E_{C}$ and $E_{N C}$.

$$
\begin{gathered}
\left(I_{E_{C}}\right)_{j k}= \begin{cases}\delta_{j k} & \text { if } e_{j} \in E_{C}, \\
0 & \text { otherwise }\end{cases} \\
\left(I_{E_{N C}}\right)_{j k}= \begin{cases}\delta_{j k} & \text { if } e_{j} \in E_{N C} \\
0 & \text { otherwise }\end{cases}
\end{gathered}
$$

Moreover, each branch equation in (2) is homogeneous to a current or to a voltage, this leads to a partition of $E$ into two subsets. The $|E| \times|E|$ matrices $\Pi_{c}$ and $\Pi_{v}$ (for currents and voltages respectively) are defined as the projectors on these two subsets.

The reduced matrices $M$ and $R$ of $\mathcal{M}$ and $\mathcal{R}$ are scaled in a consistent manner,

$$
\begin{aligned}
M^{\varepsilon} & =S_{s} M S_{v}^{-1}, \\
R^{\varepsilon} & =S_{s} R S_{c}^{-1} .
\end{aligned}
$$

The scaled reduced matrices $M^{\varepsilon}$ and $R^{\varepsilon}$ are assumed to converge towards some limit $M^{0}$ and $R^{0}$ when $\varepsilon \rightarrow 0$. If the norms $\left\|\widehat{\mathbf{i}}^{\varepsilon}\right\|,\left\|\widehat{\mathbf{v}}^{\varepsilon}\right\|,\left\|\widehat{\varphi}^{\varepsilon}\right\|[2]$ and $\left\|\widehat{\mathbf{u}}_{s}^{\varepsilon}\right\|$ are bounded then $\left(\widehat{\mathbf{i}}^{\varepsilon}, \widehat{\mathbf{v}}^{\varepsilon}, \widehat{\varphi}^{\varepsilon}, \widehat{\mathbf{u}}_{s}^{\varepsilon}\right)$ is weakly converging when $\varepsilon \rightarrow 0$ towards a limit $\left(i^{0}, v^{0}, \varphi^{0}, u_{s}^{0}\right)$ in $L^{2}(\Omega)[3]$.

The vector of electric potential $\varphi^{0}(x)$ is a constant $\varphi_{C p}^{0}(x)$ in each connected component of cell crossing pathes. So, we split it according to $\varphi^{0}=I^{0} \varphi_{C}^{0}+\varphi_{N C}^{0}$, $I^{0}$ being defined at $(32)$, and $\varphi_{N C}^{0}(x)$ being the electric potentials at nodes not in crossing pathes. In the model we refer to the vector $\varphi_{C}^{0}=\left(\varphi_{C p}^{0}\right)_{p=1, . ., n_{c}}$. Electric potential variations within connected components of crossing pathes are recovered thanks to the corrector $\varphi_{C}^{1}$ which yields the corrected electric potential field

$$
\varphi_{C}=\varphi^{0}+\varepsilon \varphi_{C}^{1} .
$$

Theorem 1 [2]

For given $\varphi_{C}^{0} \in \Psi^{H}, \Psi^{H}$ defined in (43), and $u_{s}^{0} \in$ $L^{2}(\Omega)^{|E|}$ there exist $\varphi_{C}^{1} \in L^{2}\left(\Omega ; \mathbb{R}_{\text {per }}^{|N|}\right)$ such that $\varphi_{N C}^{0} \in$ $L^{2}\left(\Omega ; \mathbb{R}^{|N|}\right), i^{0} \in L^{2}(\Omega)^{|E|}$ and $v^{0} \in L^{2}(\Omega)^{|E|}$ are solution algebraic cell circuit equations at each $x \in \Omega$,

$$
\begin{aligned}
v= & I_{E_{C}} A^{T} \varphi_{C}^{1}+I_{E_{N C}} A^{T} \varphi_{N C}^{0} \\
R^{0} i^{0}+M^{0} v= & u_{s}^{0}-M^{0}\left(\tau \nabla \varphi_{C}^{0}+I_{E_{N C}} A^{T} I^{0} \varphi_{C}^{0}\right) \\
i^{0 T} w= & 0 \\
\text { for all } w= & I_{E_{C}} A^{T} \psi_{C}^{1}+I_{E_{N C}} A^{T} \psi_{N C}^{0} \\
& \text { with }\left(\psi_{C}^{1}, \psi_{N C}^{0}\right) \in \Psi^{m}
\end{aligned}
$$

The vector $v^{0}$ is expressed by

$$
v^{0}=v+\tau \nabla \varphi_{C}^{0}+I_{E_{N C}} A^{T} I^{0} \varphi_{C}^{0} .
$$

We assume that the solution is unique. This assumption is generally satisfied once the global circuit equations has a unique solution.

The admissible nodal voltage set being

$$
\begin{aligned}
\Psi^{m}= & \left\{\left(\psi_{C}^{1}, \psi_{N C}^{0}\right) \in L^{2}\left(\Omega ; \mathbb{R}_{p e r}^{|N|}\right) \times L^{2}\left(\Omega ; \mathbb{R}^{|N|}\right)\right. \\
& \text { such that } \left.\psi_{C}^{1}=0 \text { and } \psi_{N C}^{0}=0 \text { at ground nodes }\right\}
\end{aligned}
$$

The set $\mathbb{R}_{\text {per }}^{|N|}$ is defined as,

$$
\begin{aligned}
\mathbb{R}_{p e r}^{|N|}= & \left\{\phi \in \mathbb{R}^{|N|} \text { such that } \phi_{j}=\phi_{j^{\prime}}\right. \\
& \text { for all couple } \left.\left(n_{j}, n_{j^{\prime}}\right) \text { of opposite nodes }\right\} .
\end{aligned}
$$

The $|E| \times d \times n_{c}$ tensor $\tau$ is defined by (30). We recall that $y(n) \in \mathbb{R}^{d}$ is the coordinate vector of a node $n$.

$$
\tau_{l k p}= \begin{cases}\sum_{j: n_{j} \in N_{C p}} y_{k}\left(n_{j}\right) A_{j l} & \text { for } e_{l} \in E_{C p}, \\ 0 & \text { otherwise. }\end{cases}
$$

Throughout this paper, we use the tensor product notation,

$$
(\tau \theta)_{l}=\sum_{k} \sum_{p} \tau_{l k p} \theta_{k p}
$$

where the summation is on the two last indices of $\tau$.

The $|N| \times n_{c}$ matrix $I^{0}$ is defined by

$$
I_{j p}^{0}= \begin{cases}1 & \text { if } n_{j} \in N_{C p} \\ 0 & \text { otherwise }\end{cases}
$$

$N_{C p}$ is the set of nodes involved in the branches of $E_{C p}$.

\section{Reformulation of the Problem Micro}

Theorem 2 shows that $\varphi_{C}^{0}$ is the solution of a partial differential equation, so once $\varphi_{C}^{0}$ is known, $i^{0}$ and $v^{0}$ can be computed too by theorem 1 . The equations (23-26) being linear, there exists some matrices $\mathcal{L}_{x}, \mathcal{H}_{x}$ and a third order tensor $\mathcal{P}_{x}$ such that $i^{0}, \varphi_{N C}^{0}$ and $v$ that can be expressed as function of $\varphi_{C}^{0}$, its gradient $\nabla \varphi_{C}^{0}$ and the vector source $u_{s}^{0}$,

$$
\begin{aligned}
i^{0} & =\mathcal{L}_{i} \varphi_{C}^{0}+\mathcal{P}_{i} \nabla \varphi_{C}^{0}+\mathcal{H}_{i} u_{s}^{0}, \\
\varphi_{N C}^{0} & =\mathcal{L}_{\varphi} \varphi_{C}^{0}+\mathcal{P}_{\varphi} \nabla \varphi_{C}^{0}+\mathcal{H}_{\varphi} u_{s}^{0}, \\
v & =\mathcal{L}_{v} \varphi_{C}^{0}+\mathcal{P}_{v} \nabla \varphi_{C}^{0}+\mathcal{H}_{v} u_{s}^{0} .
\end{aligned}
$$

The computation of the vector $v^{0}$ is then unchanged,

$$
v^{0}=v+\tau \nabla \varphi_{C}^{0}+I_{E_{N C}} A^{T} I^{0} \varphi_{C}^{0} .
$$




\section{Homogenized Circuit Equations (Problem Macro)} In this Section, we state the equation satisfied by $\varphi_{C}^{0}$.

\section{Theorem 2 [2]}

The vector $\varphi_{C}^{0} \in \Psi^{H}$ is the solution of the $n_{c}$ partial differential equations, so-called homogenized equations, with its boundary conditions,

$$
\begin{aligned}
A^{H}\left(\mathcal{P}_{i} \nabla \varphi_{C}^{0}+\mathcal{L}_{i} \varphi_{C}^{0}\right) & =-A^{H} \mathcal{H}_{i} u_{s}^{0}, \\
\varphi_{C p}^{0} & =0 \text { on } \Gamma_{0 p}, \\
\left(\mathcal{P}_{i} \nabla \varphi_{C}^{0}+\mathcal{L}_{i} \varphi_{C}^{0}\right) n_{\tau} & =0 \text { on } \Gamma-\Gamma_{0 p} .
\end{aligned}
$$

$\Gamma_{0 p}$ is the part of the boundary $\Gamma$ of $\Omega$ where the $p^{t h}$ connected component is grounded. The operator $A^{H}$ is defined by

$$
A^{H}=-\partial_{\tau^{*}}+I^{0 T} A I_{E_{N C}},
$$

where $\partial_{\tau^{*}} i=\tau^{*} \nabla i$ with $\tau_{p k l}^{*}=\tau_{l k p}$ and the use of notation (31). The derivative $\partial_{\tau} \varphi_{C}^{0}$ and the normal $n_{\tau}$ are defined by

$$
\begin{aligned}
\partial_{\tau} \varphi_{C}^{0} & =\tau \nabla \varphi_{C}^{0}, \\
\left(n_{\tau}\right)_{l p} & =\sum_{k=1}^{d} \tau_{l k p} n_{k},
\end{aligned}
$$

$\nabla$ being the gradient $\left(\partial_{x_{k}}\right)_{k=1 . . d}$ and $n=\left(n_{k}\right)_{k=1 . . d}$ being the outward normal vector to the boundary $\Gamma$ of $\Omega$. Remark that the coefficients $A^{H}$ and the derivatives $\nabla_{\tau}$ depends on node coordinates inherited from the expression (30) of $\tau$. Finally, the admissible set of macroscopic potential is

$$
\begin{aligned}
\Psi^{H}= & \left\{\psi \in L^{2}(\Omega)^{n_{c}} \text { such that } \partial_{\tau} \psi \in L^{2}(\Omega)^{|E|}\right. \\
& \text { and } \left.\psi_{k}(x)=0 \text { on } \Gamma_{0 k}\right\} .
\end{aligned}
$$

In the example (cf. Fig. 1), $\varphi_{C}^{0}$ has only one component $\left(n_{c}=1\right)$, we assume that $r=\varepsilon r^{0}$ and $i_{s}=\varepsilon i_{s}^{0} . \varphi_{C}^{0}$ is then solution of the partial differential equation

$$
\begin{aligned}
\frac{\partial^{2} \varphi_{C 1}^{0}}{\partial x_{1}^{2}}+\frac{\partial^{2} \varphi_{C 1}^{0}}{\partial x_{2}^{2}} & =-2 r^{0} i_{s}^{0} \text { in } \Omega, \\
\varphi_{C 1}^{0} & =0 \text { on } \Gamma_{0,1}, \\
\nabla \varphi_{C 1}^{0} \cdot n_{\tau} & =0 \text { on } \Gamma-\Gamma_{0,1} .
\end{aligned}
$$

10. Computation of Actual Voltages and Currents

Once the solution $\left(v^{0}, i^{0}\right)$ of the two-scale transform are available, actual voltages and currents may be recovered through the inverse two-scale transform (10) and inverse scaling (15-17),

$$
\begin{aligned}
& \mathbf{v} \approx T_{E}^{-1} S_{v}^{-1} v^{0} \\
& \mathbf{i} \approx T_{E}^{-1} S_{i}^{-1} i^{0} .
\end{aligned}
$$

\section{Conclusion}

The concept of two-scale transform has been detailed and illustrated in this paper. An homogenization method for periodic linear circuit based on this transform has been explained. We tried to present it in the most suitable way in view of implementation.
This method has been coded in its generality in a CAS (Computer Algebra Software). The program parses the Spice file describing the cell circuit, and generates as its output the partial differential equation of $\varphi_{C}^{0}$. This equation is then solved numerically by a FEM, and the actual voltages and currents are directly deduced.

A lot of work has still to be done: Proving the independance of the coefficients of the partial differential equation with regard to the node coordinates; Simplify the demonstration of theorem 1 (about 10 pages long); Continue to validate the method and explore its performance in terms of accuracy and computational cost.

\section{Appendices}

\section{Basic properties of some integrals on cells}

$$
\begin{aligned}
\int_{Y_{\mu}^{\varepsilon}} d x^{\prime} & =\varepsilon^{d} \\
\int_{Y_{\mu}^{\varepsilon}} \chi_{Y_{\lambda}^{\varepsilon}}\left(x^{\prime}\right) d x^{\prime} & =\varepsilon^{d} \delta_{\mu \lambda}
\end{aligned}
$$

\section{Derivation of the expression of $T_{E}^{*}$}

$$
\begin{aligned}
& \left(T_{E} \mathbf{v}, u\right) \\
= & \int_{\Omega}\left(T_{E} \mathbf{v}\right) \cdot u(x) d x \\
= & \sum_{j=1}^{|E|} \int_{Y_{\mu}^{\varepsilon}}\left(T_{E} \mathbf{v}\right)_{j}(x) \cdot u_{j}(x) d x \\
= & \sum_{\mu \in\{1, . ., m\}^{d}} \sum_{j=1}^{|E|} \varepsilon^{-d} \int_{Y_{\mu}^{\varepsilon}} \chi_{Y_{\mu}^{\varepsilon}}(x) u_{j}(x) d x V_{\mu j} \\
= & \varepsilon^{d} \sum_{\mu \in\{1, . ., m\}^{d}} \sum_{j=1}^{|E|} \varepsilon^{-d} \int_{Y_{\mu}^{\varepsilon}} u_{j}(x) d x V_{\mu j} \\
= & \varepsilon^{d}\left(T_{E}^{*} u\right)^{T} \cdot \mathbf{v} \\
= & \varepsilon^{d} \sum_{\mu \in\{1, . ., m\}^{d}} \sum_{j=1}^{|E|}\left(T_{E}^{*} u\right)_{\mu j} V_{\mu j} \\
\Rightarrow & \left(T_{E}^{*} u\right)_{\mu j} \\
= & \varepsilon^{-d} \int_{Y_{\mu}^{\varepsilon}} u_{j}(x) d x
\end{aligned}
$$

Proof that $T_{E}^{*} T_{E}=I_{\mathcal{E}}$ on $\mathbb{R}^{|\mathcal{E}|}$

Let $\mathbf{u} \in \mathbb{R}^{|\mathcal{E}|}$ and $\mathcal{I} \sim(\mu, j)$,

$$
\begin{aligned}
\left(T_{E}^{*} T_{E} \mathbf{u}\right)_{\mathcal{I}} & =T_{E}^{*}\left(\sum_{\lambda \in\{1, . ., m\}^{d}} U_{\lambda j} \chi_{Y_{\lambda}^{\varepsilon}}(x)\right) \\
& =\varepsilon^{-d} \int_{Y_{\mu}^{\varepsilon}} \sum_{\lambda \in\{1, . ., m\}^{d}} \chi_{Y_{\lambda}^{\varepsilon}}(x) d x U_{\lambda j} \\
& =\varepsilon^{-d} \varepsilon^{d} U_{\mu j} \\
& =\mathbf{u}_{\mathcal{I}}
\end{aligned}
$$




$$
\begin{aligned}
\text { Proof that } T_{E} T_{E}^{*}=I_{E} \text { on } \mathbb{P}^{0}(\Omega)^{|E|} & \\
& \text { Let } u \in \mathbb{P}^{0}\left(\Omega ; \mathbb{R}^{|E|}\right), \\
& \left(T_{E} T_{E}^{*} u\right)_{j}(x) \\
= & \left(T_{E}\left(\varepsilon^{-d} \int_{Y_{\mu}^{\varepsilon}} u_{j}\left(x^{\prime}\right) d x^{\prime}\right)\right)_{j}(x) \\
= & \sum_{\mu \in\{1, . ., m\}^{d}} \varepsilon^{-d} \int_{Y_{\mu}^{\varepsilon}} u_{j}\left(x^{\prime}\right) d x^{\prime} \chi_{Y_{\mu}^{\varepsilon}}(x) \\
= & \sum_{\mu \in\{1, . ., m\}^{d}} \sum_{\lambda \in\{1, . ., m\}^{d}} \varepsilon^{-d} \int_{Y_{\mu}^{\varepsilon}} \chi_{Y_{\lambda}^{\varepsilon}}\left(x^{\prime}\right) d x^{\prime} U_{\lambda j} \chi \chi_{Y_{\mu}^{\varepsilon}}(x) \\
= & \sum_{\mu \in\{1, . ., m\}^{d}} \sum_{\lambda \in\{1, . ., m\}^{d}} \delta_{\mu \lambda} U_{\lambda j} \chi_{Y_{\mu}^{\varepsilon}}(x) \\
= & \sum_{\mu \in\{1, . ., m\}^{d}} U_{\mu j} \chi_{Y_{\mu}^{\varepsilon}}(x) \\
= & u_{j}(x)
\end{aligned}
$$

\section{Acknowledgements}

This work has been supported by the research project CNRS-PEPS 2007-2009 "Prototype for a software dedicated to Atomic Force Microscope Arrays" and by the EC project "PIDEA EUROSCIL" under contract \#06-2-930193.

\section{References}

1. L.O. Chua, A.D. Desoer and S.K. Kuh, "Linear and Nonlinear Circuits", McGraw-Hill Series in Electrical Engineering, 1987.

2. M. Lenczner, "Homogenization of linear spatially periodic electronic circuits", Networks and Heterogeneous Media, 1 (2006), no. 3, pp. 467-494.

3. K. Yosida, "Functional Analysis", Classic in Mathematics, Springer Verlag, 1995. 